\title{
APPLICATION OF MULTIPLE CATEGORIES OF UNMANNED AIRCRAFT SYSTEMS (UAS) IN DIFFERENT AIRSPACES FOR BUSHFIRE MONITORING AND RESPONSE
}

\author{
N. Homainejad ${ }^{\text {a }}$, C. Rizos ${ }^{a} *$ \\ ${ }^{a}$ The University of New South Wales, School of Civil and Environmental Engineering, Sydney, NSW, 2052, Australia \\ n.homainejad@student.unsw.edu.au,c.rizos@unsw.edu.au
}

Commission VI, WG VI/4

KEY WORDS: Bushfire, UAS, Category, Endurance, Imaging Sensor, Communication System, Mission Planning

\begin{abstract}
:
Demand and interest in Unmanned Aircraft Systems (UAS) for civilian applications, and advances in technology such as development of sense-and-avoid systems, will soon allow UAS to be flown alongside manned aircrafts in non-segregated airspace. An area that can benefit from the application of UAS is the bushfire services sector. Currently such services rely on watchtowers, fixed-wing manned aircrafts and satellite data for reliable information. UAS are a promising alternative to traditional methods of collecting bushfire data. There are several varieties of UAS and each category has certain limitations, hence a combination of multiple UAS with features appropriate for bushfire emergencies can be used simultaneously for collecting valuable data. This paper will describe the general UAS categories, some characteristics of Australian bushfires, and speculate on how a combination of several UAS operating in different airspaces can be of benefit for bushfire response personnel and firefighters.
\end{abstract}

\section{INTRODUCTION}

In the past decade, with advances in technology and price reductions, the civilian applications of Unmanned Aircraft Systems (UAS) has grown significantly, making data acquisition more convenient and accessible for a wide range of users (Jensen et al., 2010; Wang et al., 2008). Originally driven primarily by military use, UAS have begun finding application among civilian users for earth sensing reconnaissance and scientific data collection purposes (Watts et al., 2012). An area where the application of UAS has been recognised as valuable is bushfire or wildfire mapping monitoring, management and combat.

Australia's environmental conditions make it one of the most fire-prone countries in the world, and a large percentage of the population reside or work in high-risk areas, creating an ongoing need for better firefighting capabilities. To combat bushfires effectively, early detection and continuous tracking is vital (Casbeer et al., 2005). Due to the hazardous nature of bushfires firefighters ideally require near real-time information about the area to conduct an effective and safe firefighting mission (Graml and Wigley, 2008). Currently firefighters collect information on the state of a fire through a combination of satellite, aerial and ground observation. While each source has advantages, such as breadth for satellite imagery, resolution for aerial imagery, and obliqueness for ground-based imagery, there are also limitations (Adams et al., 2010; Chou et al., 2010). Satellite imagery suffers from lack of spatial resolution and timely availability of data due to orbit restrictions denying emergency services real-time data. On the other hand, not only are manned aircraft costly but crew duties limit aircraft mission to 4-10 hours, and they are unable to operate during dangerous weather conditions (Adams et al., 2010; Grenzdörffer et al., 2008; Xu et al., 2014).
The rapid development of UAS technology has enabled greater use as photogrammetry and remote sensing platforms to complement satellite and aerial remote sensing systems (Ezequiel et al., 2014). Because of their flight performance, high image resolution, adaptability to fly at different altitudes and ability to access remote and dangerous environments, UAS can overcome most limitations of traditional methods of obtaining aerial and remote sensing imagery. All the advantages listed above, and others, make UAS the perfect data acquisition platform for firefighters during bushfire missions.

This paper will make recommendations on the most suitable UAS categories for bushfire missions. This includes looking at features such as endurance, sensor payload, communication system (data link), weight, and resistibility of the UAS to wind. A further objective will be to consider how to coordinate the simultaneous operation of different categories of UAS for effective bushfire mission planning and response management.

\section{UAS DESIGN AND AUSTRALIA'S PATH}

The Global Air Traffic Management Operational Concept defines an Unmanned Aircraft System as (ICAO, 2011):

"An unmanned aerial vehicle is a pilotless aircraft, in the sense of Article 8 of the Convention on International Civil Aviation, which is flown without a pilot-in-command on-board and is either remotely and fully controlled from another place (ground, another aircraft, space) or programmed and fully autonomous."

UAS comprise of the following components: a remotely piloted aircraft (RPA), Ground Control Station (GCS), C2 (command and control) data link and a remote pilot (RP). An UAS can be operated semi-autonomous, autonomously, or a combination of both by an RP from a Ground Control Station (GCS). 


\subsection{UAS Terminology}

Different taxonomies can be used to define an UAS. With the term Unmanned Aerial Vehicle (UAV) now obsolete, familiar and synonymous terminologies that are used, including Remotely Piloted Aircraft System (RPAS), Pilotless Aerial Vehicle (PAV) and Unmanned Aircraft (UA). CASA, the Civil Aviation Safety Authority of Australia has been quick to acknowledge international UAS terminology standardisations by the International Civil Aviation Organisation (ICAO), and has replaced the term Unmanned Aerial Vehicle (UAV) with the more correct term, Unmanned Aircraft Systems (UAS) or Remotely Piloted Aircraft (RPA) and Remotely Piloted Aircraft System (RPAS), as appropriate (CASA, 2014a, 2014b). CASA has also recognised the stigma that comes with the label 'drone' and has been quick to identify this term as a misleading imprint in the public domain (CASA, 2013).

\subsection{Limitations}

Currently UAS face two types of limitations, technical and regulatory. Technical limitations are a result of an immature technology and may ultimately be addressed through rapid technological development. These limitations can impact on the response time and the activity, but can be partially overcome by selecting a suitable UAS for the activity in mind. Regulatory limitations are rules and regulations set nationally or by ICAO. The principle objective of the regulatory framework is safety.

Despite the limitations UAS face at the present time, developments in UAS technology for civilian and commercial applications continue, with predictions that the Small category of UAS, generally referred to as small Unmanned Aircraft Systems (sUAS), will be the fastest growing category of UAS for civilian and commercial operations. This is due to their great versatility and relatively low cost and low operating expenses.

\subsection{Australia's UAS Legislations}

CASA was quick to notice the growing commercial interest in UAS. As a result CASA produced the Advisory Circular (AC 101-1(0)) as guidance for the safe manufacture and operation of UAS in Australia.

The role of CASA is to oversee the safe and legal operations of UAS in Australia by eliminating/minimising hazards to persons and property aloft or on the ground that should not pose a greater danger than that of equivalent manned aircraft, class or category. The operating standards include:

- Visual Line of Sight (VLOS) - direct visual contact of the RPA by the remote crew without the aid of spectacles

- Operational altitude of below $400 \mathrm{ft}$ above ground or water

- $\quad$ No flights within populous areas, including a $30 \mathrm{~m}$ separation gap between any bystander not involved in the operation and the aircraft

- Day Visual Meteorological Conditions (VMC)

- Minimum of 3 nautical mile (NM) of separation from all aerodromes

- Operate outside of controlled airspace

- Operate outside of prohibited, restricted and dangerous areas

\section{UAS CATEGORISATION}

The UAS primary airframe type is either fixed-wing or rotary. They vary considerably in their size, flight endurance and other capabilities, and thus can be categorised into a number of different classes (Ameri et al., 2009; Everaerts, 2008; Watts et al., 2012). CASA has classified RPAS into the following categories based on their size (CASA, 2002):

- $\quad$ Micro RPA - RPA with gross weight of 100 grams or less

- $\quad$ Small RPA - RPA with a gross weight of $2 \mathrm{~kg}$ and below

- $\quad$ Medium RPA - RPA with a gross weight greater than $2 \mathrm{~kg}$ and less than or equal to $150 \mathrm{~kg}$

- $\quad$ Large RPA - RPA with a gross weight greater than $150 \mathrm{~kg}$

Although no recognised international UAS category system exists, UAS classification based on military descriptions in terms of size, flight endurance and capabilities, is described by (Watts et al., 2012). This paper will adopt (Watts et al., 2012) UAS category terminology:

- $\quad$ MAV/ NAV (Micro-UAV/ Nano-UAS)

- $\quad$ VTOL (Vertical Take-Off and Landing)

- $\quad$ LASE (Low Altitude, Short Endurance)

- $\quad$ LALE (Low Altitude, Long Endurance)

- MALE (Medium Altitude Long Endurance)

- HALE (High Altitude Long Endurance)

\subsection{MAV/ NAV (Micro-UAS/ Nano-UAS}

This category of UAS evolved as a result from the US military's interest in developing miniature spy devices. They are small in size and operate at very low altitudes, below $300 \mathrm{~m}$. They are limited in flight time and sensor payload, and applications are not useful for bushfire missions. Therefore there will be no further reference to this category of UAS in this paper.

\subsection{VTOL (Vertical Take-Off and Landing)}

As fixed-wing UAS become larger in size, they can no longer be launched by hand and require a runway for take-off and landing. VTOL UAS have the advantage of having hovering capabilities and manoeuvrability, and are an obvious alternative to fixed-wing UAS. They are not limited to a runway for takeoff and landing, and therefore are of particular utility in remote areas or disaster regions where there may be no suitable aerodrome or runway.

This category of UAS comes in different sizes and can fly at different altitudes but at present their small size is their most popular characteristic. Smaller scale VTOL UAS have a limited payload, require rechargeable batteries and carry miniaturised sensors. Developers of conventional aircraft have to take into account payload weight, and hence range of operations. On smaller scale UAS these requirements are even more restrictive. An example of this category of UAS is the AscTec Falcon 8 (Figure 1)

\subsection{LASE (Low Altitude, Short Endurance)}

As their name implies this category of UAS is low endurance, low in cost and can fly within Visual Line of Sight for between 45 minutes to 2 hours at altitudes up to $450 \mathrm{~m}$. This category of 
UAS is unstable, especially in windy weather and to compensate for this disadvantage flight plans for aerial photography are designed in the direction of the wind with larger forwards $(80 \%)$ and side $(60 \%)$ overlaps. The sensor payload is also limited; the system is generally capable of collecting and storing all the data on board, on an SD card. An example of this category would be the MAVinci SIRIUS (Figure 1).

Sensor limitations, short flight endurance, their relatively light weight and their inability to provide real-time bushfire data makes this category of UAS impractical for bushfire services. Continuously changing bushfire environments require (near) real-time monitoring of the bushfire environment.

\subsection{LALE (Low Altitude Long Endurance)}

LALE UAS fall in the higher end of the small UAS (sUAS) category. They can carry larger payloads, of the order of a few $\mathrm{kg}$ at altitudes of up to $5000 \mathrm{~m}$. As their name implies, this category of UAS can fly for extended periods of 20 hours or longer, many kilometres away from the GCS. An example is the Boeing Insitu ScanEagle (Figure 1).

Although this category of UAS has limitations, such as a weak communication data link, they also have many advantages. This category of UAS is long endurance. They typically collect and provide georeferenced colour, black and white imagery, and/or black and white IR videos. This is particularly useful for firefighters as it can aid bushfire response management.

\subsection{MALE (Medium Altitude Long Endurance) UAS}

This category of UAS is predominantly intended for military applications and is much larger in size than the previous categories. They have more advanced aerodynamic design and control systems due to their high operational requirements, and can operate to altitudes of $9000 \mathrm{~m}$ and above. This category of UAS can be sent on flights hundreds of kilometres from their ground stations, on missions lasting many hours (20-40 hours) (Watts et al., 2012). Examples of this category of UAS are the NASA Ikhana Predator-B and IAI Heron (Machatz-1) (Figure $1)$.

MALE UAS can be deployed within a short time and are capable of supplying near real-time geo-rectified, multi-spectral imagery to firefighters. Their high payload capability allows them to carry a variety of sophisticated sensors including electro-optical (EO), infrared (IR) and synthetic aperture radar (SAR). This makes them ideal not only for bushfire operations but for imaging for a wide range of disaster scenarios.

\subsection{HALE (High Altitude Long Endurance) UAS}

HALE UAS are large and complex, and are capable of operating as a "very low-orbit" satellite by remaining in free airspace above $14 \mathrm{~km}$ for days, weeks or even months (Fransaer et al., 2004). Anything between 12 and $25 \mathrm{~km}$ altitude falls within the stratospheric region. The advantage of the stratospheric region is that air traffic is controlled up to $14 \mathrm{~km}$ altitude, and above that height an aircraft is not limited in its movements, and in fact there is virtually no air traffic, allowing for more efficient mission planning (Everaerts et al., 2004).

This category of UAS is also capable of resolving the inherent drawbacks of both aerial and satellite platforms when equipped with appropriate sensors, as it can observe any area, for significant periods of time (Everaerts et al., 2004; Fransaer et al., 2004). An example of this category is the Northrop Grumman RQ-4 Global Hawk (Figure 1).
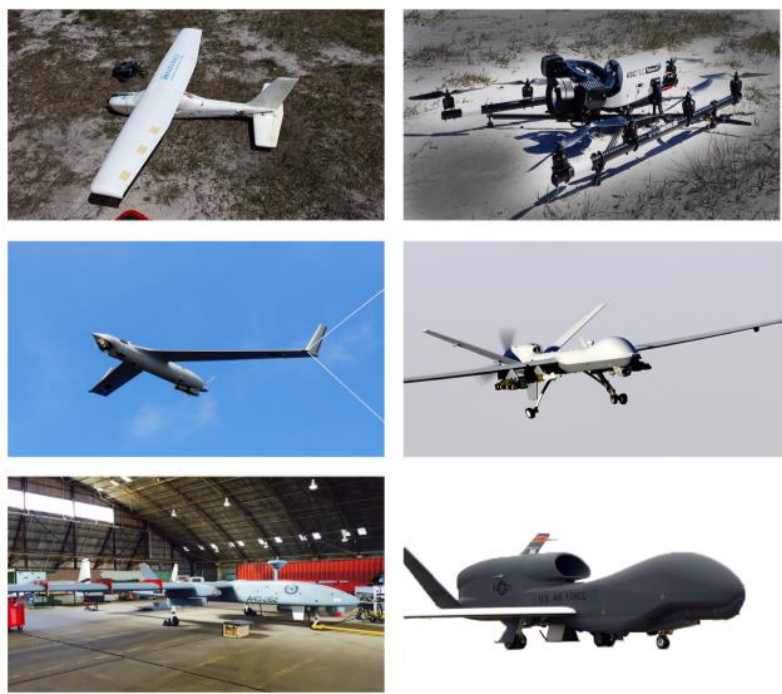

Figure 1- From left to right, top to bottom: MAVinci SIRIUS, AscTec Falcon 8, Boeing Insitu ScanEagle, NASA Ikhana Preditor B, IAI Heron (Machatz-1) courtesy of Royal Australian Air Force (RAAF), Northrop Grumman RQ-4 Global Hawk

\section{BUSHFIRES IN AUSTRALIA AND THE APPLICATION OF UAS FOR BUSHFIRE MISSIONS}

Bushfires in Australia are an inevitable natural disaster due to Australia's vast, hot and generally dry landscape, coupled with unpredictable climatic conditions. Bushfires can pose serious threats to lives, properties, communities, and place a large economic burden on the Australian economy. An extensive study by News Corp Australia revealed that in the past 13 years bushfires have caused about $\$ 2.6$ billion in damages (Cornish, 2014). The three factors that determine whether a bushfire will occur include the presence of fuel, oxygen, terrain, wind and an ignition source. The presence of fuel and variations in wind speed and direction cause unpredictable changes in fire patterns and intensity.

Fire-rate and fire intensity can change within a short period of time. The influencing factors that influence the acceleration of fires include the moisture content of fuel, large volumes of dead fuels and to a lesser extent, living vegetation. Additional factors include fuel surface area, distribution of fuel in the vertical plane, combustion rate, and burnout times of fuel, atmospheric instability including humidity, ground wind speed, terrain slope and the "spotting" process. The time of day also plays a role as the factors that increase the rate of fires are linked to the diurnal regime of wind, humidity and temperature.

Currently a number of methods are used for bushfire detection and to plan responses. These include watchtowers, fixed-wing aircraft and satellite remote sensing. The data collected can be analysed to coordinate rescue efforts, disaster response, identify access points and verify experimental disaster modelling (Jensen et al. 2009). Different methods are incorporated for different stages relative to the occurrence of a fire: 
1. Fire prevention - which involves identifying risks and taking necessary actions to reduce damage impacts.

2. Monitoring fire activity and response to combating the disaster.

3. The recovery phase - fire assessment after the burning.

\subsection{Sensors}

The two most distinguishing features of a natural fire, particularly a luminous one, are its apparent source temperature and the power spectral density of the radiation intensities emitted from it (Sivathanu and Tseng, 1997). Whilst the electro optical (EO) wavelength can be used during day time aerial reconnaissance, the IR portion of the electromagnetic spectrum is the most useful wavelengths for day and night bushfire reconnaissance. The Mid-infrared (wavelengths $3 \mu \mathrm{m}-5.5 \mu \mathrm{m}$ ) region is sensitive to radiation emitted from objects of high temperature, of the order of $800 \mathrm{~K}$ to $1000 \mathrm{~K}$, and is used to detect strong radiation emissions from fire fronts whilst the farinfrared (wavelengths $8 \mu \mathrm{m}-14 \mu \mathrm{m}$ ) region is used to detect the naturally emitted radiation due to the Earth's temperature. These two bands are used together because the hypersensitivity of the mid-infrared band on its own can cause channel saturations (Dionizio and Trinder, 2012). Furthermore, NIR (near infrared) spectral band can be used to provide accurate data by eliminate false- positive, spectrally bright objects detected during the day.

\subsection{Research and Development on the Application of UAS for Bushfire Missions}

A short review of the application of the different categories of UAS utilised for bushfire missions is presented below.

(Casbeer et al., 2005) demonstrated the use of multiple LASE UAS for bushfire missions. An effective UAS path planning algorithm utilising infrared images collected on-board in realtime while flying along the boundary of the fire was developed, and while promising had limitations. The author was quick to note limitations such as initial rendezvous time, dealing with fuel contingencies and refuelling, and operations during irregular and growing fire shapes.

In 2013 the ScanEagle UAS, which was originally designed for military applications, was used by Australian firefighters in the Wollemi National Park fires. This long endurance UAS provided day and night surveillance and reconnaissance information to firefighters. The imagery from the ScanEagle was used to assess the movement of the fire front and to identify the locations of high-risk spot fires. This flight trial showcased to Australian firefighters how the application of military grade UAS can be utilised by emergency services for bushfire monitoring and response, while enhancing the safety of first responders. The application of military grade UAS for disaster relief and response will continue to evolve in Australia, with the Royal Australian Air Force (RAAF) announcing that they have entered a Memorandum of Agreement (MoA) with Airservices Australia, an Australian Government-owned air navigation service provider, for operating the Heron UAS in Australian civil airspace (Defence, 2015).

The NASA Ikhana a Predator-B UAS was used during 20072009 for wildfire surveillance in the western United States. It was the first civilian UAS to receive a Certificate of Airworthiness from the FAA (Federal Aviation Authority) to be able to operate in U.S National Airspace System for disaster support and wildfire event imaging (Watts et al., 2012). The Ikhana is a sophisticated UAS equipped with duplicate sensors carried on orbiting satellites, including EO sensors to provide colour video and IR images for night vision (Merlin, 2009). It could also carry a SAR payload plus laser designations, spotting and range-finding systems and was successfully used in 20 missions (60 fires) (Merlin, 2009). The versatility of the sensors makes it a multi-purpose UAS capable of being used for different mission scenarios. The satellite communications on the Ikhana UAS allows the RP to operate and steer the RPA. This also allows the UAS to provide near-real-time, geo-rectified imagery to incident management teams within 10 minutes of acquisition, allowing an improved understanding of fire conditions to permit optimal deployment of firefighting resources.

\section{MULTIPLE UAS MISSION PLANNING FOR BUSHFIRE EVENTS}

Continuously changing fire patterns make ground-based reconnaissance difficult and dangerous. Hence for this purpose aerial reconnaissance is preferred. Although satellite and manned aircrafts have advantages they also have many limitations. UAS can compensate for these limitations. A method to overcome the current limitations and to exploit UAS for their full capabilities is to use different categories of UAS in different airspaces in such a way that each category of UAS is capable of substituting the current tasks of satellite and manned aircrafts for bushfire missions (Figure 2).

To be able to undertake a reliable, productive and safe mission a well-designed UAS flight plan is essential. This includes a careful design of aircraft trajectory, with built-in flexibility, and real-time mission management of the RPA. This is significant, especially when organising multiple UAS missions. MALE or HALE UAS categories can be used as alternate sources of satellite-like data as they are capable of flying at higher altitudes and can carry larger, more sophisticated sensor payloads. These UAS are not only capable of supplying remotely sensed data but they are capable of providing (near-) real-time georeferenced data (within 10-15min) to firefighters. In regions close to airports or populated areas HALE category UAS may be a preferred option compared to the MALE category, as they can avoid disruption to air traffic movements in non-segregated airspace. LALE category UAS can be used at a lower altitude concurrently with MALE or HALE categories. By flying at lower altitudes LALE UAS are capable of providing a close-up view of an area of interest. For example, this category of UAS can be used for aerial survey of escape routes, properties or used for fire spotting, which is valuable information that is difficult to identify from high altitude imagery. Firefighters can use this data for ground mission planning.

A HALE or MALE category UAS could be flown with a LALE category UAS, and the two categories can follow a similar flight plan, with the lower altitude UAS over-flying an area a few minutes behind the higher altitude UAS. This will allow the two UAS to supply different categories of data over the same region from different altitudes without the lower altitude UAS overlapping in the higher altitude UAS scan. Another approach is to have the LALE category UAS flying along the fire boundary, supplying firefighters with critical aerial surveys to prevent firefighters from placing their lives in unnecessary danger. The data collected can be uploaded to, for example, Google Earth to provide firefighters with a "global" perspective 
of the fire, and can be used with recent satellite data for further analyses. The different sets of data can be used to derive different levels of information about the region of interest, with the higher altitude data used for hotspot analysis while the lower altitude data is used for aerial survey and reconnaissance of properties, escape routes and any further information that can assist firefighters.

To be able to operate a multi-mission UAS, the UAS should have features suited for monitoring bushfire behaviour. Multiple UAS operations require the following:

- Appropriate sensors (navigation sensors, imaging sensors).

- High frequency and high bandwidth, command, control and communication data link.

- Long endurance flight capability.

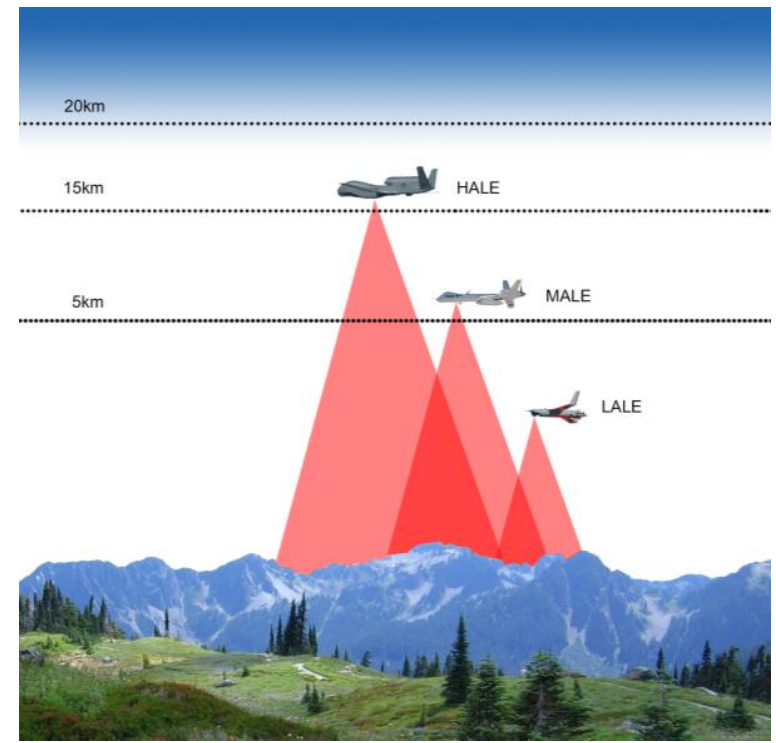

Figure 2 -Multiple UAS flight scenario

\subsection{Sensor Payload and Resolution}

The key challenge in the use of UAS for bushfire missions is to convert the data collected into reliable information. Hence the choice of sensor and the sensor resolution are important. Larger UAS can carry a heavier sensor payload. They are capable of carrying sophisticated sensors similar to those on satellites. Imaging payloads may consist of electro-optical (EO), infrared (IR) and synthetic aperture radar (SAR). Lighter UAS categories, such as the LALE category can carry EO and IR imaging sensors. Using different categories of UAS simultaneously makes current sensor and sensor resolution issues redundant, with each category mostly compensating for the sensor and sensor resolution limitations of the other UAS.

\subsection{Endurance}

Long endurance UAS categories are more suitable for "dull and dirty" bushfire missions and are capable of operating throughout the day and night. This category of UAS is useful for continuously changing fire patterns as there is no down-time in retrieving and refuelling the UAS. Not only do these UAS carry sophisticated navigation and imaging sensors, they can also supply firefighters with continuous georeferenced data. Long endurance UAS are safer to operate compared to short endurance UAS. They also ensure that the RP and crew are a safe operating distance from the fire front.

\subsection{Communication Systems}

UAS data links can be divided into two categories: RF Line-ofSight (RF LOS) and Beyond Line-of-Sight (BLOS). An UAS data link consists of a radio frequency transmitter and receiver, an antenna and a modem to link all these components with the RPA sensors. BLOS data link contains LOS technologies along with satellite-based communications (SATCOM). SATCOM is for long endurance UAS that fly well beyond line-of-sight (e.g. $200-600 \mathrm{~km})$. SATCOM is used for C2 of the RPA and for communications (C3) with Air Traffic Control. Different SATCOM frequency bands are possible, with the most common being $\mathrm{Ku}$ band, $\mathrm{K}$ band, $\mathrm{S}$ band, $\mathrm{L}$ band, and $\mathrm{C}$ band, with the $\mathrm{X}$ band mainly reserved for military use. Frequency bands are particularly important for long endurance bushfire missions. As the distance between the GCS and the RPA increases, the signal-to-noise ratios of the transmission link will fall. The signal loss may manifest as a lag in the RPA command uplink and recovery of data downlink.

\section{CONCLUSION}

Earth observation techniques can assist firefighters in developing and executing improved bushfire management strategies. They can aid in reducing the impact of a bushfire by providing firefighters with critical details of the location, extent and main access routes to the disaster zone. However traditional methods of using satellites and manned aircraft have limitations.

UAS have become increasingly popular as photogrammetric and remote sensing platforms for civilian use. They address many of the limitations of satellite and manned aircraft data with their high image resolution, high accuracy, near to real-time imagery. To be able to exploit UAS for their full capacity, and to overcome limitations of different UAS, a combination of different categories deployed in different airspaces can be used for bushfire missions. The higher altitude data can be used for hot-spot analysis while the lower altitude data is used for aerial surveys and reconnaissance by first responders. The key for a successful multiple UAS mission is a well-designed mission plan. UAS features that should be taken into account for multiple UAS bushfire mission are endurance, data link(s) and sensors. Implementing a multiple UAS mission could improve data acquisition and analysis for more effective bushfire management.

\section{ACKNOWLEDGEMENTS}

We acknowledge WGCDR Jonathan McMullan and Heron 1 MALE RPAS operator, Janelle Sheridan from the Royal Australian Air Force (RAAF) for supplying photos of the IAI Heron (Machatz-1), MALE category UAS.

\section{REFERENCES}

Adams, S., Friedland, C., Levitan, M., 2010. Unmanned Aerial Vehicle Data Acquisition for Damage Assessment in Hurricane Events, Proceedings of the 8th International Workshop on Remote Sensing for Disaster Management, Tokyo, Japan.

Ameri, B., Meger, D., Power, K., Gao, Y., 2009. UAS

Applications: Disaster \& Emergency Management. American Society for Photogrammetry and Remote Sensing, pp. 45-55. 
CASA, 2002. Unmanned Aircraft and Rockets Unmanned Aerial Vehicle (UAV) Operations, Design Specification, Maintenance and Training of Human Resources. CASA, Australia.

CASA, 2013. RPAs (Drones) in Civil Airspace and Challenges for CASA, A public Seminar Organised by the Sir Richard Williams Foundation. Civil Aviation Safety Authority, Government of Australia, Canberra, Australia HTTP://www.casa.gov.au/scripts/nc.dll?WCMS:STANDARD: pc=PC_101593 (08 Aug. 2014).

CASA, 2014a. Notice of Proposed Rule Making, Remotely Piloted Aircraft Systems, NPRM 1309OS - May 2014. CASA, Canberra, Australia.

CASA, 2014b. Annex C Draft Advisory Circulars:101-1, 101-4, 101-5. CASA, Australia.

Casbeer, D.W., Beard, R.W., McLain, T.W., Sai-Ming, L., Mehra, R.K., 2005. Forest fire monitoring with multiple small UAVs, 2005 American Control Conference, ACC, June 8, 2005 - June 10, 2005. Institute of Electrical and Electronics Engineers Inc., Portland, OR, United States, pp. 3530-3535.

Chou, T.-Y., Yeh, M.-L., Chen, Y.-C., Chen, Y.-H., 2010. Disaster monitoring and management by the unmanned aerial vehicle technology, ISPRS Technical Commission VII Symposium on Advancing Remote Sensing Science, July 5, 2010 - July 7, 2010. International Society for Photogrammetry and Remote Sensing, Vienna, Austria, pp. 137-142.

Cornish, L., 2014. Bushfires in Australia deadlier, more destructive and worse to come, News Limited. http://www.news.com.au/technology/environment/bushfires-inaustralia-deadlier-more-destructive-and-worse-to-come/storye6frflp0-1226815740178 (01 Mar. 2015).

Defence, 2015. Airservices Australia and Defence strengthen collaboration on Unmanned Aircraft Systems, Departmnet of Defence, Australian Government, Canberra, ACT, http://news.defence.gov.au/2015/05/29/airservices-australiaand-defence-strengthen-collaboration-on-unmanned-aircraftsystems/ (01 Jun. 2015).

Dionizio, M., Trinder, J.C., 2012. Preliminary Design of a Small Satellite Constellation for Monitoring Bushfires in Australia. ISPRS Journal of Photogrammetry and Remote Sensing, Melbourne, Australia.

Everaerts, J., 2008. The use of unmanned aerial vehicles (UAVs) for remote sensing and mapping. The International Archives of the Photogrammetry, Remote Sensing and Spatial Information Sciences 37, 1187-1192.

Everaerts, J., Lewyckyj, N., Fransaer, D., 2004. Pegasus: design of a stratospheric long endurance UAV system for remote sensing. The International Archives of the Photogrammetry, Remote Sensing and Spatial Information Sciences 35.

Ezequiel, C.A.F., Cua, M., Libatique, N.C., Tangonan, G.L., Alampay, R., Labuguen, R.T., Favila, C.M., Honrado, J.L.E., Canos, V., Devaney, C., 2014. UAV aerial imaging applications for post-disaster assessment, environmental management and infrastructure development, Unmanned Aircraft Systems (ICUAS), 2014 International Conference on. IEEE, pp. 274283.

Fransaer, D., Lewyckyj, N., Vanderhaegehen, F., Everaerts, J., 2004. PEGASUS: Business model for a stratospheric long endurance UAV system for remote sensing. The international archives of the photogrammetry, remote sensing and spatial information sciences, Istanbul, Turkey.

Graml, R., Wigley, G., 2008. Bushfire hotspot detection through uninhabited aerial vehicles and reconfigurable computing, Aerospace Conference, 2008 IEEE. IEEE, pp. 1-13.

Grenzdörffer, G.J., Engel, A., Teichert, B., 2008. The photogrammetric potential of low-cost UAVs in forestry and agriculture. The International Archives of the Photogrammetry Remote Sensing and Spatial Information Sciences 31, 12071214.

ICAO, 2011. Unmanned Aircraft Systems (UAS), ICAO Circular 328-AN/190 Quebec, Canada.

Jensen, A.M., Morgan, D., Chen, Y.Q., Clemens, S., Hardy, T., 2010. Using multiple open-source low-cost unmanned aerial vehicles (UAV) for 3D photogrammetry and distributed wind measurement, International Design Engineering Technical Conferences and Computers and Information in Engineering Conference. American Society of Mechanical Engineers, San Diego, CA, USA, pp. 629-634.

Merlin, P.W., 2009. Ikhana: Unmanned Aircraft System Western States Fire Missions. National Aeronautics and Space Administration, NASA History Office, Washington, D.C. USA

Sivathanu, Y.R., Tseng, L.K., 1997. Fire detection using time series analysis of source temperatures. Fire Safety Journal 29, 301-315.

Wang, J., Garratt, M., Lambert, A., Wang, J.J., Hana, S., Sinclair, D., 2008. Integration of GPS/INS/Vision Sensors to Navigate Unmanned Aerial Vehicles. The International Archives of the Photogrammetry, Remote Sensing and Spatial Information Sciences XXXVII, 863-970.

Watts, A.C., Ambrosia, V.G., Hinkley, E.A., 2012. Unmanned aircraft systems in remote sensing and scientific research: Classification and considerations of use. Remote Sensing 4, 1671-1692.

Xu, Z., Yang, J., Peng, C., Wu, Y., Jiang, X., Li, R., Zheng, Y., Gao, Y., Liu, S., Tian, B., 2014. Development of an UAS for post-earthquake disaster surveying and its application in Ms7.0 Lushan Earthquake, Sichuan, China. Computers and Geosciences 68, 22-30. 